\title{
Transport of Multiply and Highly Charged Ions through Nanoscale Apertures in Silicon Nitride Membranes
}

\author{
F.I. Allen ${ }^{\mathrm{a}, 1, *}$, A. Persaud ${ }^{\mathrm{a}}$, S.J. Park ${ }^{\mathrm{a}}$, A. Minor ${ }^{\mathrm{a}}$, M. Sakurai ${ }^{\mathrm{b}}$, D.H. Schneider ${ }^{\mathrm{c}}$, and T. Schenkel ${ }^{\mathrm{a}}$ \\ ${ }^{a}$ Lawrence Berkeley National Laboratory, Berkeley, CA 94720, USA \\ 'Physics Department, Kobe University, 1-1 Rokko-dai, Nada, Kobe 657-8501, Japan \\ ${ }^{\mathrm{c}}$ Lawrence Livermore National Laboratory, Livermore, CA 94550, USA \\ ${ }^{1}$ Institut für Physik der Humboldt-Universität zu Berlin, Lehrstuhl Plasmaphysik, Newtonstraße 15, \\ D-12489 Berlin, Germany
}

We report on experimental studies of the transport of multiply $\left(\mathrm{Ar}^{2,3+}, 0.2-0.3 \mathrm{keV} / \mathrm{u}\right)$ and highly charged ions $\left(\mathrm{Ar}^{16+}, \mathrm{Xe}^{4++}, 2-3 \mathrm{keV} / \mathrm{u}\right)$ through nanoscale apertures. Arrays of apertures with diameters of 50 to $200 \mathrm{~nm}$ and aspect ratios of 1.5:1 to 1:5 were formed in silicon nitride membranes by focused ion beam drilling in combination with ion beam assisted thin film deposition. The fraction of ions undergoing charge exchange is found to be in agreement with model predictions for low charge state ions, while it is much lower than expected for high charge state ions. We discuss results in relation to a classical over-the-barrier model and capillary guiding mechanisms.

PACS: 34.50. Dy

Keywords: Slow highly charged ions; Capillary transmission; Charge exchange

${ }^{1}$ Permanent address

*Corresponding author. Tel.: +49-30-2093-7546; fax: +49-30-2093-7531; e-mail: allen@physik.hu-berlin.de 


\section{Introduction}

Studies of the interactions of slow ( $\left.\mathrm{v}<\mathrm{v}_{\text {Bohr }}\right)$, multiply and highly charged ions with solids aim at gaining a fundamental understanding of ion-solid interaction mechanisms, and at the development of techniques for materials modification and surface analysis [1,2]. Transport of ions through nanometer scale apertures is crucial for the integration of ion beams with scanning probes, a technique which is being developed for local doping of materials [3].

The interaction of slow, multiply and highly charged ions with (metallic and insulating) surfaces has been described very successfully in a classical over-the-barrier model (COB) [4-7]. The model can be used to estimate the critical distance for charge transfer $d_{c} \approx \sqrt{2 q} / W_{\Phi}$ (in atomic units), where $\mathrm{q}$ is the charge of the projectile ion and $\mathrm{W}_{\Phi}$ is the work function of a metal surface. When a planar target is replaced by an aperture or a capillary [7-10], several trajectories can be distinguished. Ions can pass through the capillary without charge exchange, ions can be attracted to the capillary walls, collide, and get implanted into the wall, or ions can be attracted to the capillary walls reaching the critical distance for electron capture and emerge having undergone charge exchange. Recently, a process of capillary guiding was reported [9] where ions are reflected from a capillary wall by a repulsive potential without undergoing charge exchange. On analysis and detection of all projectiles which transmit the capillary, the fraction of charge exchange can be determined, and a comparison with model predictions can be made.

In this article we present results for charge exchange of multiply and highly charged ions for transmission of apertures in silicon nitride targets. Benchmark data for the transmission of ions through apertures in insulating and metallic materials are important for ion projection lithography and the development of ion implantation with scanning probe alignment. In the latter, ions are transported through a single nanoscale aperture in an atomic force microscope (AFM) tip [3]. The results presented in this paper are from arrays of several hundred holes in order to increase the surface area available for charge exchange.

\section{Experimental}

Low stress silicon nitride membranes (200 and $500 \mathrm{~nm}$ thick) on silicon frames were sputter coated with a thin film $(\sim 20 \mathrm{~nm})$ of a gold-palladium alloy to prevent charging under ion and electron beam exposure. Holes of diameter 100 to $300 \mathrm{~nm}$ were drilled into the membranes using an FEI Strata 235 focused ion beam (FIB) microscope at the National Center for Electron Microscopy at Lawrence Berkeley National Laboratory. This tool combines a low divergence, highly focused $\mathrm{Ga}^{+}$ion beam with a scanning electron microscope (SEM) column. Arrays of up to 800 holes were drilled using a $30 \mathrm{keV} \mathrm{Ga}^{+}$beam with an intensity of $10 \mathrm{pA}$ and a beam spot size of about $10 \mathrm{~nm}$. Holes were imaged in situ using the SEM (Fig. 1).

The maximum aspect ratio achievable using the FIB technique on the membranes was about 5:1. This limit results from a balance of sputtering and re-deposition of target material. For high aspect ratios, re-deposition dominates, precluding the direct drilling of deep, narrow holes. Holes with smaller diameters than achievable by direct drilling alone can be formed via electron and ion beam assisted thin film deposition. Here, a precursor gas, e.g. an organometallic compound containing platinum or the gas tetraethylorthosilicate (TEOS), are introduced into the process chamber. Secondary electrons - emitted where the primary ion or electron beams strike the surface crack the precursor gas, leading to deposition of thin films. The hole diameter is gradually reduced when a thin film is deposited across a hole [11]. This standard technique was used here to close 100 $\mathrm{nm}$ diameter holes down to a residual diameter of $50 \mathrm{~nm}$.

Highly charged ions of argon and xenon were produced with an Electron Beam Ion Trap (EBIT, [12]) and a Refrigerated Electron Ion Beam Trap (REBIT, [13]). A low density plasma ion 
source was used to produce $\mathrm{Ar}^{1+, 2+, 3+}$ ions. After extraction with potentials of 2-12 kV the ions were steered and focused with a series of electrostatic deflectors and Einzel lenses in the beam line (base pressure $10^{-8}$ Torr). A $90^{\circ}$ bending magnet was used to select a particular charge state according to its mass-to-charge ratio. The beam was then collimated to a spot size of $3 \mathrm{~mm}$, and a divergence of about $0.8^{\circ}$. The pressure in the target chamber was $10^{-8}$ Torr.

Fig. 2 shows a schematic of the experimental setup [1]. The target membranes were mounted inside a Faraday cup. The various charge states of the emerging ions were separated using an electrostatic analyzer placed $35 \mathrm{~cm}$ downstream of the membrane targets. The analyzer consisted of two parallel plates biased typically at $+/-100 \mathrm{~V}$. A position sensitive microchannel plate detector with a resistive anode, placed $45 \mathrm{~cm}$ past the parallel plate analyzer was used for detection of transmitted projectiles. Projectile flight times from the membranes to the analyzer ranged from 0.4 to $1.5 \mu \mathrm{s}$, a long duration compared to expected lifetimes of excited states formed in charge exchange processes. This experiment thus probes the projectile charge state distribution after transmission of apertures, and following a long equilibration time in vacuum.

\section{Results and Discussion}

Fig. 3 shows a lineout of data from the position sensitive detector for the transmission of $\mathrm{Ar}^{3+}$ ions through $100 \mathrm{~nm}$ diameter holes in a $500 \mathrm{~nm}$ thick silicon nitride membrane. The total fraction of transmitted ions undergoing charge exchange was $3.7 \%$. The neutral fraction is found to be slightly higher than the fraction of $\mathrm{Ar}^{1+}$ ions, in agreement with previous observations $[7,8]$. Relatively low ion dose rates precluded us from observing an effect of exposure time on charge exchange.

In Fig. 4 we show the total charge exchange fraction for $\mathrm{Ar}^{2+}$ ions and a series of aperture sizes. The results are shown together with the data point for $\mathrm{Ar}^{3+}$ from Fig. 3 and a previous result for a single $1.5 \mu \mathrm{m}$ diameter hole in a chromium AFM tip [14]. The fraction of ions undergoing charge exchange increases with decreasing aperture size. The effective work function of silicon nitride can be estimated from the sum of its electron affinity $(\sim 5 \mathrm{eV})$ and band gap $(\sim 2 \mathrm{eV})$ to be about $7 \mathrm{eV}$. With this estimate, we can calculate the radius for electron capture, and the expected charge exchange fraction from the ratio between the capture radius and the aperture diameter, $2 \mathrm{~d}_{\mathrm{c}} / \mathrm{r}$, according to the classical over-the-barrier model. We include the expected charge exchange fraction in Fig. 4. A second curve is added, calculated with a work function of $5 \mathrm{eV}$ representing electron capture from a metal, i.e. from the platinum or gold-palladium coatings or the chromium of the AFM tip. The data points for $\mathrm{Ar}^{2+}$ and silicon nitride follow the model predictions for a target surface with a $7 \mathrm{eV}$ work function qualitatively. Evidently, the work function of surfaces in these apertures is not well know since several materials of not well-defined composition and structure were used, and the experiments were not performed in ultra high vacuum.

For $\mathrm{Ar}^{16+}$ and $\mathrm{Xe}^{44+}$, charge exchange fractions in the interaction with $200 \mathrm{~nm}$ diameter apertures in silicon nitride are predicted at $\sim 2.3$ and $\sim 3.8 \%$, respectively. Fig. 5 shows a line out for $\operatorname{Ar}^{16+}(2 \mathrm{keV} / \mathrm{u})$ transmitted through $200 \mathrm{~nm}$ diameter holes in a $500 \mathrm{~nm}$ thick silicon nitride membrane. At the given sensitivity of our experimental setup, we did not observe charge exchange for either $\mathrm{Ar}^{16+}$ or $\mathrm{Xe}^{44+}$. The experimental upper limit of charge exchange is $0.4 \%$ for $\mathrm{Ar}^{16+}$, and $3 \%$ for $\mathrm{Xe}^{44+}(3 \mathrm{keV} / \mathrm{u}$, not shown).

The marked suppression of charge exchange for transport of $\mathrm{Ar}^{16+}$ through apertures in the insulator might be due to charging of the aperture walls, similar to a recently reported capillary guiding mechanism [9]. Here, ion strikes of aperture walls are associated with electron emission, where secondary electron emission yields increase roughly linear with the ion charge state. The resulting distribution of holes in the insulating silicon nitride leads to the buildup of a repulsive wall 
potential for wall areas close to the entrance and exit sides of the apertures. The localized build up of positive charges on the surface then repels incident ions, preventing ions from reaching the critical distance for electron capture. More experimental studies and theoretical modeling are needed to clarify this mechanism in a regime of high charge state ions, relatively low ion dose rates, and for varying aperture materials (e.g. insulating membranes with or without metal film coatings).

Exposure of the hole arrays to highly charged ions could have resulted in their enlargement due to electronic sputtering $[1,2]$. A competing process of hole closing by ad-atom diffusion is also reported to occur under specific conditions of target temperature, composition and ion dose rate [15]. Hence, after experimental runs which typically involved 1 to 20 hours of beam exposure with ion currents of pA (highly charged ions) to nA (lower charge states) with $3 \mathrm{~mm}$ diameter beam spots, the hole arrays were inspected by SEM. No change in the aperture structure was observed by SEM imaging and we conclude that significant target erosion or ad-atom diffusion did not occur.

\section{Summary}

Investigations into the amount of charge exchange on transmission of multiply and highly charged ions through a series of nanoscale apertures of varying sizes in silicon nitride are reported. In the case of the multiply charged ions, charge exchange fractions are in qualitative agreement with predictions from a classical over-the-barrier model. Charge exchange is found to be suppressed for highly charged ions, indicating a possible mechanism of wall guiding.

\section{Acknowledgements}

This work was supported by the U.S. Department of Energy under Contract No. DEAC0376SF00098. The first author would also like to thank the Max-Planck-Institut für Plasmaphysik, Germany and the EURATOM Association. Further, we would like to thank Joseph McDonald for his support with the REBIT. 


\section{References}

[1] T. Schenkel, A.V. Hamza, A.V. Barnes, D.H. Schneider, Prog. Surf. Sci. 61 (1999) 23

[2] F. Aumayr, H.P. Winter, Phil. Trans. R. Soc. Lond. A 362 (2004) 77

[3] A. Persaud, S.J. Park, J.A. Liddle, J. Bokor, I.W. Rangelow, T. Schenkel, Nano Lett. 5 (2005) 1087

[4] J. Burgdörfer, P. Lerner, F.W. Meyer, Phys. Rev. A 44 (1999) 5647

[5] L. Hägg, C.O. Reinhold, J. Burgdörfer, Phys. Rev. A 55 (1997) 2097

[6] J.J. Ducrée, F. Casali, U. Thumm, Phys. Rev. A 57 (1998) 338

[7] K. Tőkési, L. Wirtz, C. Lemell, J. Burgdörfer, Phys. Rev. A 64 (2001) 042902

[8] S. Ninomiya, Y. Yamazaki, F. Koike, H. Masuda, T. Azuma, K. Komaki, K. Kuroki, M.

Sekiguchi, Phys. Rev. Lett. 78 (1997) 4557

[9] N. Stolterfoht, J.-H. Bremer, V. Hoffmann, R. Hellhammer, D. Fink, A. Petrov, B. Sulik, Phys. Rev. Lett. 88 (2002) 133201

[10] T. Schenkel, I.W. Rangelow, R. Keller, S.J. Park, J. Nilsson, A. Persaud, V.R. Radmilovic, P. Grabiec, D.H. Schneider, J.A. Liddle, J. Bokor, Nucl. Instr. and Meth.B 219-220 (2004) 200

[11] T. Schenkel, V. Radmilovic, E.A. Stach, S.J. Park, A. Persaud, J. Vac. Sci. Technol. B 21 (2003) 2720

[12] T. Schenkel, A. Persaud, A. Kraemer, J.W. McDonald, J.P. Holder, A.V. Hamza, D.H. Schneider, Rev. Sci. Instrum. 73 (2002) 663

[13] http://www.physicstechnology.com/

[14] A. Persaud, F.I. Allen, F. Gicquel, S.J. Park, J.A. Liddle, T. Schenkel, Tzv. Ivanov, K.

Ivanova, I.W. Rangelow, J. Bokor, J. Vac. Sci. Technol. B 22 (2004) 2992

[15] J. Li, D. Stein, C. McMullan, D. Branton, M.J. Aziz, J.A. Golovchenko, Nature 412 (2001) 166 


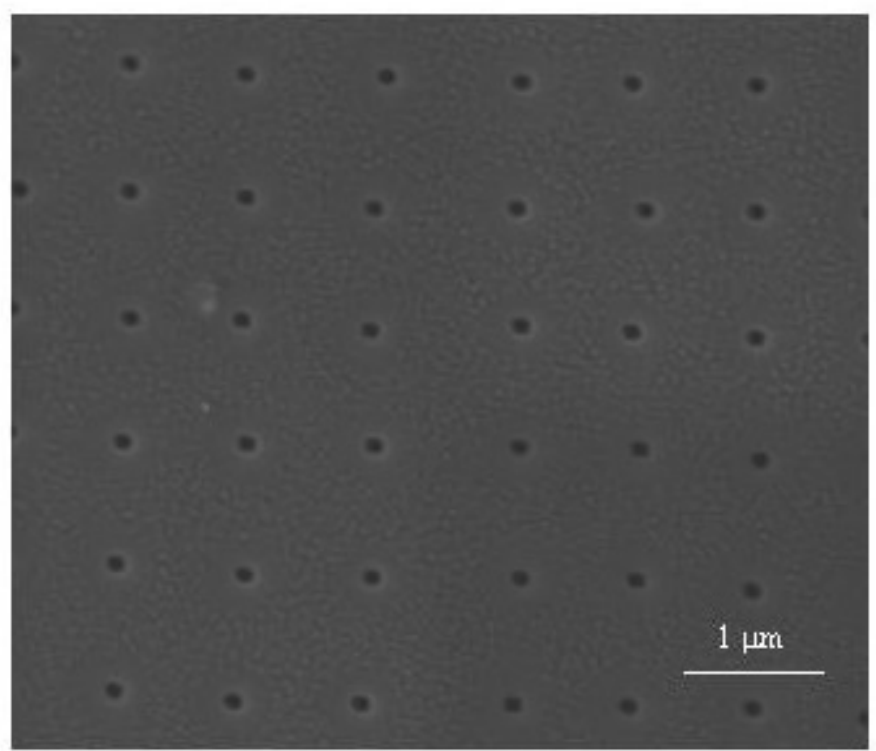

Fig. 1. SEM image of part of an array of $100 \mathrm{~nm}$ diameter holes in a $500 \mathrm{~nm}$ thick silicon nitride membrane. Light and dark areas show contrast due to the gold-palladium coating. 


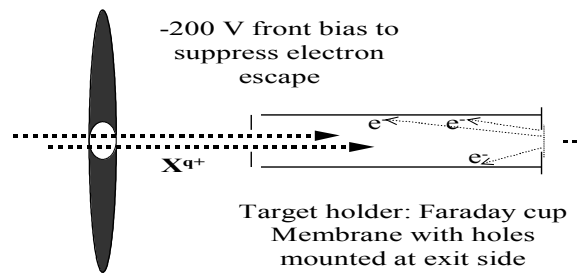

Pre-collimator

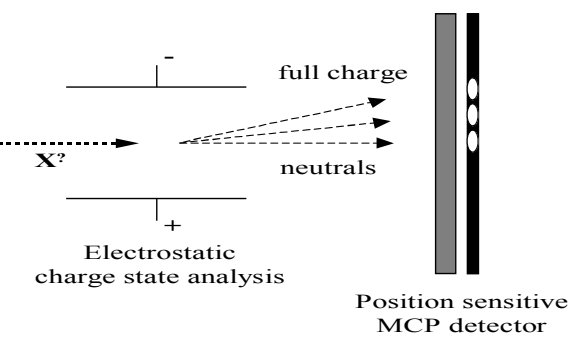

Position sensitive
MCP detector

Fig. 2. Schematic of the setup for charge exchange measurements. 


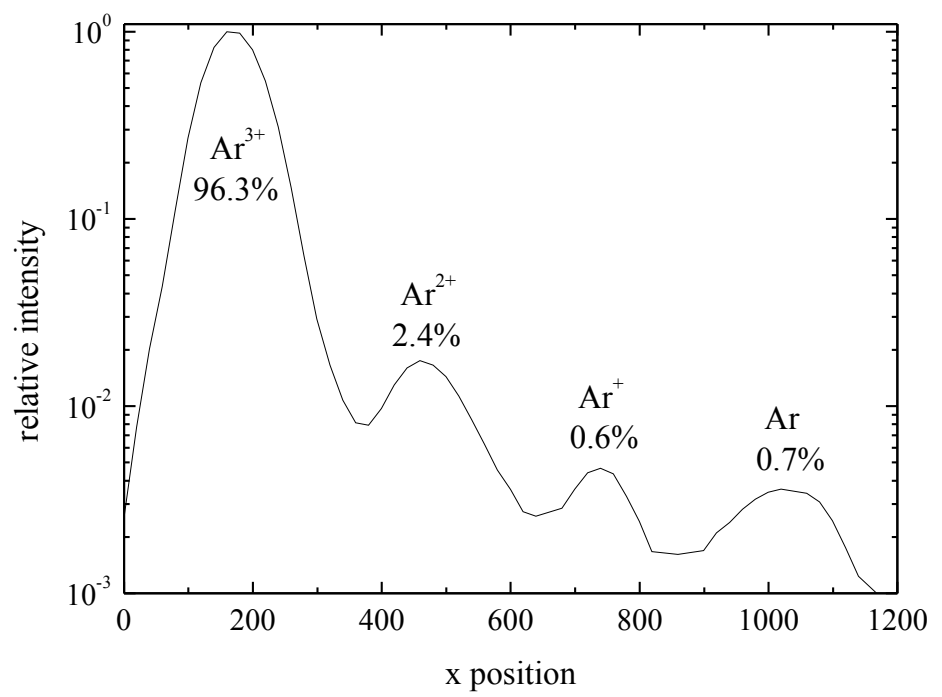

Fig. 3. Charge state fractions of argon ions after transmission of $0.3 \mathrm{keV} / \mathrm{u} \mathrm{Ar} \mathrm{r}^{3+}$ through $100 \mathrm{~nm}$ diameter holes in a $500 \mathrm{~nm}$ thick silicon nitride membrane. 


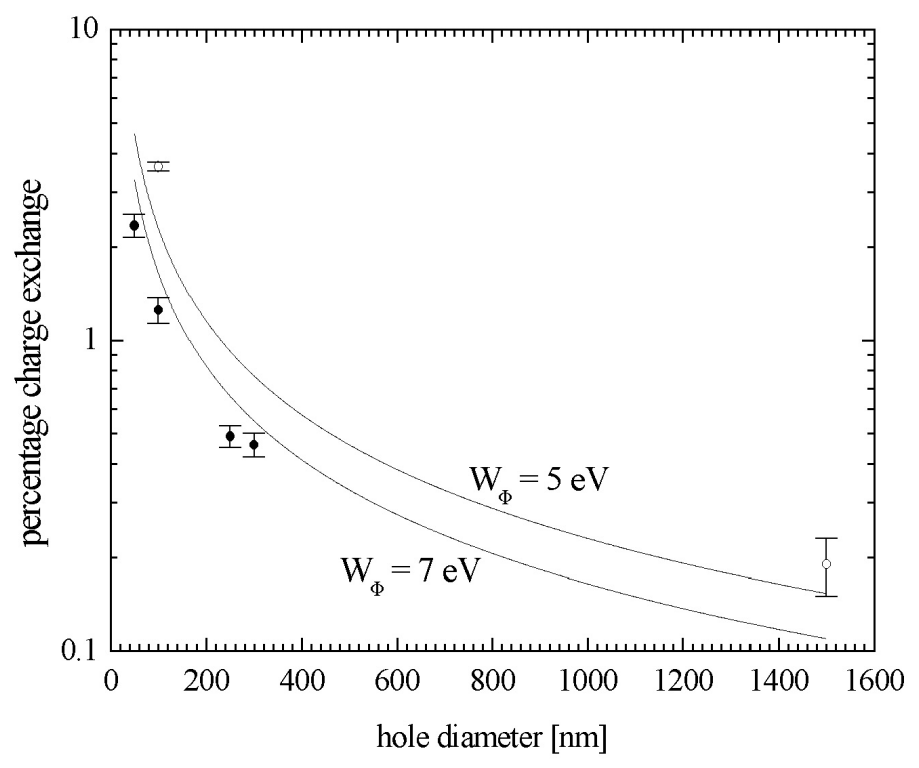

Fig. 4. Total amount of charge exchange versus aperture size for $0.2 \mathrm{keV} / \mathrm{u} \mathrm{Ar}{ }^{2+}$ ions (200 $\mathrm{nm}$ thick silicon nitride membranes, solid dots). The $50 \mathrm{~nm}$ hole size was achieved by ion beam assisted platinum deposition on $100 \mathrm{~nm}$ holes. The open data point at $100 \mathrm{~nm}$ corresponds to $0.3 \mathrm{keV} / \mathrm{u} \mathrm{Ar}{ }^{3+}$

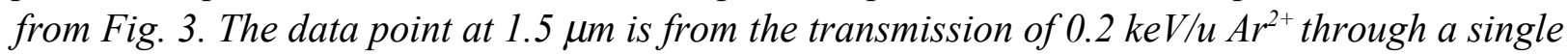
hole in a chromium AFM tip [14]. The curves show predictions from a classical over-the-barrier model for two work functions. 


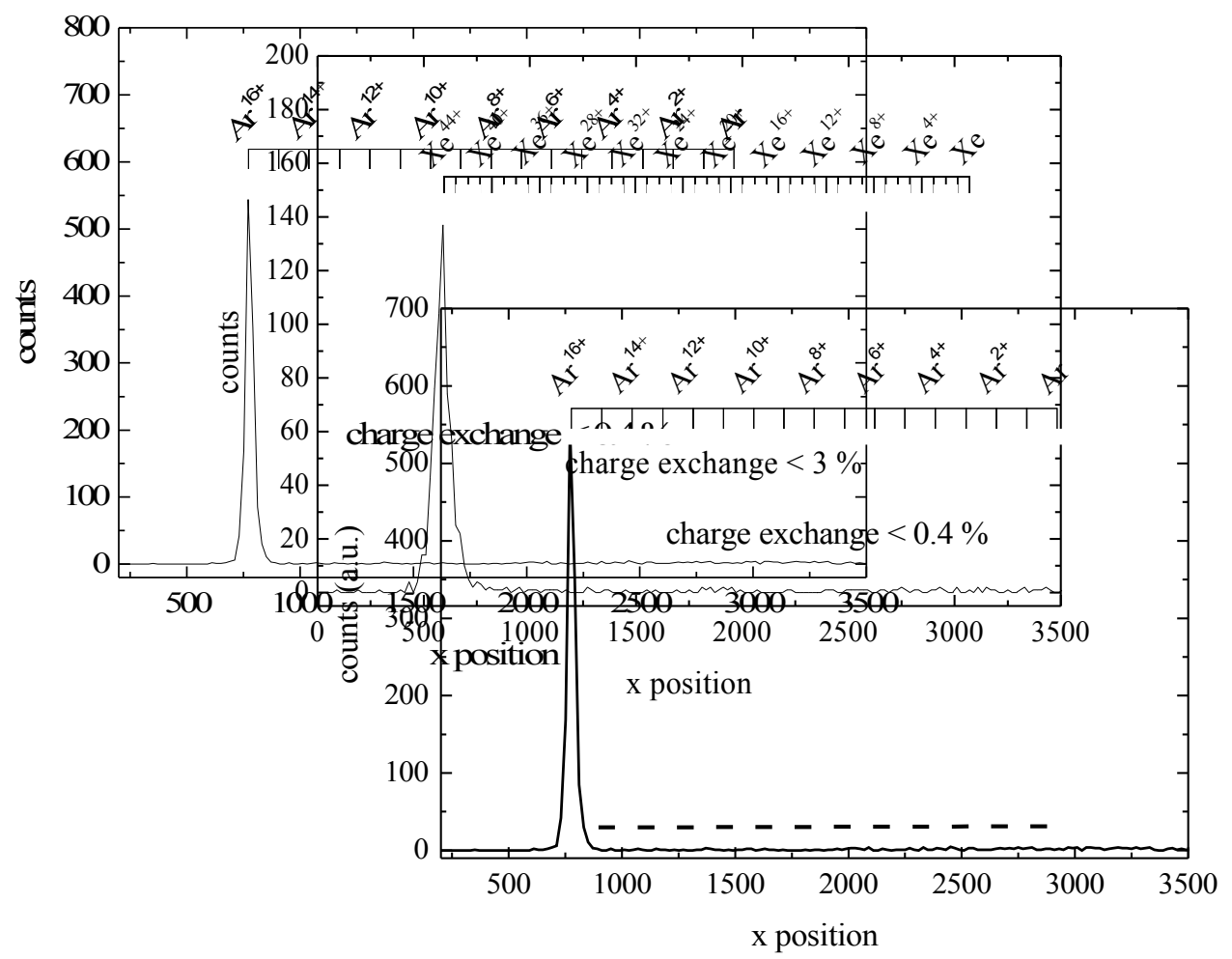

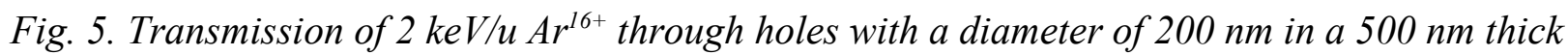
silicon nitride membrane. The upper limit for charge exchange is $0.4 \%$ compared to $2.3 \%$ expected for processes described in a classical over-the-barrier model (indicated by the dashed line). 